\title{
Producción de semilla sexual bajo las condiciones tropicales de Nicaragua
}

\section{Fidel Torres*, Alma Olivas**}

\begin{abstract}
RESUMEN
Experimentos en producción de semilla sexual de papa (SSP) realizados durante cinco años en diferentes zonas de Nicaragua ubicadas entre los $900 \mathrm{y}$ 1,400 msnm, muestran la factibilidad de producir en esta región tropical, SSP de buen vigor germinativo, rendimiento de tubérculos y en cantidades suficientes para satisfacer la demanda nacional de semilla. En las zonas altas del departamento de Estelí ubicadas entre los 1,300 y 1,400 msnm, durante los meses de mayo-agosto, en los que se registran promedios de temperaturas máximas/mínimas de $27^{\circ} \mathrm{C} / 15^{\circ} \mathrm{C}$, temperaturas del suelo de $20^{\circ} \mathrm{C}, 13 \mathrm{hr}$ de luz y precipitaciones de $300 \mathrm{~mm}$ durante dicho período, han mostrado ser las mejores condiciones locales para la producción de SSP. En 1991 se produjeron $5 \mathrm{Kg}$. de SSP de polinización libre (PL) empleando clones selectos; semilla de los clones Serrana INTA, CFK-69.1, LT- 7 y Atzimba mostraron mayor o similar vigor que algunas progenies híbridas producidas por CIP e India, y obtuvieron rendimientos de tubérculos-semillas en almacigos de 9.1,7.4,6.7 y $6.1 \mathrm{Kg} . \mathrm{m}^{2}$ respectivamente. En 1992 se produjo $7.12 \mathrm{Kg}$. de SSP híbrida de los cruzamientos Serrana x LT-7 (2.46 Kg.) y Serrana x 104.12 LB (4.66 Kg.) con aplicaciones adicionales de N (240 Kg./ha) y de N más P (240 Kg. N/ ha + 600 Kg. P/ha) durante la floración de las plantas madres. A los 105 días de almacenamiento, las semillas de Serrana x 104.12 LB y Serrana x LT-7 de las plantas madre que recibieron suministro excedente de $\mathrm{N}+\mathrm{P}$, mostraron mayor porcentaje de germinación (99.0\% y 95.0\%) y el coeficiente de velocidad CoV (20.7 y 20.5) que aquellas cuyas plantas madre recibieron sólo $\mathrm{N}$ adicional (87.3\% y 76.8\% de germinación; 19.7y 19.2 de CoV). El costo de producción por Kg. de Serrana x LT-7 fue de \$750 dólares US y de Serrana x 104.12 LB de \$400.
\end{abstract}

Palabras claves adicionales: producción de SSP, híbridos, polinización libre, progenitores, trópico cálido, vigor.

Aceptado para publicación: abril 26, 1993

* Biólogo, Coordinador de Generación y Transferencia de Tecnología del Programa Nacional de Papa (PNP) Ministerio de Agricultura y Ganadería (MAG). Región I; Estelí Nicaragua.

* * Agrónomo Asistente de Investigación, PNP, Estelí Nicaragua. 


\section{SUMMARY}

\section{Production of Sexual Seeds of Potato under Tropical Conditions of Nicaragua}

Experiments on production of sexual seeds of potato conducted during 1987 1992 in different regions of Nicaragua located between 900 and 1,400 masl demonstrated the possibility to produce sufficient quantity of high quality seeds in Nicaragua to satisfy the national demand for potato seed. The best local conditions to produce seed were found to be in the higher altitude regions of Estelí (>1,300 masl) during May-August, in which air temperatures range from 27 to $15{ }^{\circ} \mathrm{C}$ and average soil temperature in $20 \mathrm{C}$ with 13 hours of day length and $300 \mathrm{~mm}$ of rain.

In 1991, the clones Serrana, CFK-69.1, LT-7 and Atzimba produced $5 \mathrm{~kg}$ of open pollinated seed. The seed quality was superior or similar to the hybrid progenies produced at CIP and in India. In 1992,7.12 kg of high quality sexual seeds of Serrana x LT-7 and Serrana x 104.12 LB were produced with additional nitrogen applications. The production cost per $1 \mathrm{~kg}$ was 750 and 400 US dollars, respectively.

Additional Index words: production of TPS, hybrids, open pollination, parents, warm tropics, vigor.

El uso de semilla sexual de papa (SSP) o semilla en la producción de papa de Nicaragua es una realidad en proceso de desarrollo. Durante 1992 se han sembrado 80 ha de papa con tubérculos provenientes de SSP y obtenido rendimientos de 20 a 25 t/ha (23). Su aceptación en el mercado y el surgimiento de productores especializados en el uso de SSP que producen intensivamente tubérculos-semillas en diferentes épocas del año para abastecer la demanda de pequeños agricultores de escasos recursos, constituyen bases seguras de la adopción de esta tecnología de producción de papa. La continuidad de su expansión dependerá de la disponibilidad de SSP, de buena adaptación y rendimiento, así como de cantidades suficientes, según la demanda de los agricultores. El desarrollo de técnicas eficientes para producir SSP de calidad a bajo costo es de primera importancia para asegurar su disponibilidad; sin embargo, en los países del tercer mundo, ubicados en los trópicos, esto constituye una barrera importante $(5,6,10)$.

El rol del ambiente es de particular importancia en la producción de SSP vigorosa (14), la intensidad de la floración y la formación de bayas en papa son favorecidas por días largos (16 a $17 \mathrm{hr}$ ) y bajas temperaturas $\left(15\right.$ a $18{ }^{\circ} \mathrm{C}$ 
en promedio). La producción de SSP en climas cálidos es dificultosa debido que los días son cortos (12-13 hr) y el rango de temperaturas es de media a alta (20 a $\left.28{ }^{\circ} \mathrm{C}\right)(1)$; sin embargo, cuando se usan clones seleccionados para ser usados como parentales bajo condiciones de alta temperatura, la producción de semilla mejora considerablemente, la dormancia es menor y la adaptabilidad a las altas temperaturas es mayor que la semilla producida en climas frios (10).

En muchos países tropicales, hay una creciente demanda de los productores por SSP de calidad. Países de Asia, África y Centroamérica han expresado sus dificultades para producir SSP debido a condiciones climáticas desfavorables. Se han obtenido resultados promisorios en la identificación de materiales parentales apropiados y prácticas culturales para una eficiente producción de semilla sexual en áreas tropicales (3). Aspectos importantes son la evaluación del potencial de las posibles zonas en las que se puede producir SSP, la elevación del nivel nutricional de las plantas madres para optimizar el potencial genético de la semilla durante la germinación y su establecimiento temprano, mediante aplicaciones suplementarias de nitrógeno durante la floración (13); como también de fósforo, que en otras hortalizas como, zanahoria, berro y lechuga, ejercen efecto positivo en el vigor de la semilla producida $(17,20,24,9,16)$; especialmente en cultivos de semillas diminutas con escasa reserva de fosfatos que, por lo común, es insuficiente para un crecimiento enérgico (24).

En el presente trabajo se ha estudiado la producción de SSP bajo las condiciones tropicales de Nicaragua, el efecto de dos formas de suministro de $\mathrm{N}$ y $\mathrm{P}$ a las plantas madres sobre la cantidad, peso y vigor de la semilla producida y se compara el vigor y rendimiento de SSP producida localmente con SSP proporcionada por CIP e India.

\section{MATERIALES Y MÉTODOS}

\section{Experimento 1: Producción de semilla sexual de polinización libre en Nicaragua, La Almaciguera; 1350 msnm, 1991.}

Este experimento se realizó en la finca "San Francisco", localidad La Almaciguera (1350 msnm 12.8 $\mathrm{N}$ ), Estelí; durante el período junioseptiembre de 1991, los clones parenteales empleados fueron: Tollocan, 7 XY. 1, LT-7, Serrana INTA, Atlantic, Atzimba, DTO-28, DTO-33, CFK-69.1 y 104-12LB provenientes del Centro Internacional de la Papa (CIP) y multiplicados en la localidad de El Naranjo (900 msnm) durante mayo-agosto de 1990. Los clones se plantaron el 30 de mayo, en estado de brotación múltiple en surcos de $20 \mathrm{~m}$ de longitud, $1.2 \mathrm{~m}$ de distancia entre surcos y $0.3 \mathrm{~m}$ entre plantas; se dispusieron en grupos de dos parentales que se plantaron asociados en surcos alternos, con 4 surcos para cada parental: Tollocan/7XY. 1, LT7/Serrana INTA, LT- II Atlantic, Atzimba/Atlantic, DTO-28/ DTO-33, DTO28/Atlantic, CFK-69.1/Atzimba, 104.12 LB/CFK-69.1. La fertilización que recibieron las plantas al momento de la siembra fue de $150-300-180 \mathrm{~kg} / \mathrm{ha}$ de 
N-P-K.

La aplicación adicional de N (urea) a las plantas madres fue de $240 \mathrm{~kg} / \mathrm{ha}$ en seis fraccionamientos cada 8 días, aplicado en banda a $10 \mathrm{~cm}$ de distancia de las plantas, a partir del momento de la aparición de los primeros botones florares. Debido a que no se contaba con un número homogéneo de tubérculos de cada parental los bloques no tuvieron repeticiones. Las plantas no fueron aporcadas y se sostuvieron con estacas y cuerdas para evitar el acamado. Las condiciones de crecimiento de las plantas se caracterizaron por días de $13.0 \mathrm{hr}$ de longitud, temperaturas promedio máximas/mínimas de 27 ${ }^{\circ} \mathrm{C} / 17{ }^{\circ} \mathrm{C}$ y precipitación de $250 \mathrm{~mm}$ durante todo el período fenológico; se evitó el ataque de plagas y enfermedades durante el periodo vegetativo, floración y fructificación. La actividad de los polinizadores durante la floración fue intensa especialmente de Bombas spp. Las bayas se cosecharon aproximadamente a las 6 semanas del inicio de la polinización debido a la senescencia de las plantas, aunque las bayas no estaban reblandecidas. Las bayas fueron fermentadas por $48 \mathrm{hr}$ para facilitar la extracción de la semilla que fue desinfectada con una solución de hipoclorito de sodio al $0.5 \%$ por tres minutos, se secó a temperatura ambiental de $21{ }^{\circ} \mathrm{C}$ y almacenó sobre arroz tostado como desecante en termos de plástico a temperatura ambiental promedio de $24{ }^{\circ} \mathrm{C}$ durante 6 meses. El peso de semilla cada progenie PL producida se registró tomando 5 muestras de 100 semillas de cada una utilizando una balanza analítica electrónica digital. La evaluación del vigor germinativo de las semillas producidas se hizo a los seis meses después de su extracción; primero en platos petri, colocando 100 semillas por cada progenie con cinco repeticiones, esta prueba se repitió dos veces; posteriormente se realizó una prueba de campo en almácigos de suelo franco como sustrato, la unidad experimental fue de $1 \mathrm{~m}^{2}$ para cada progenie, distancia de siembra de 10 x $10 \mathrm{~cm}$ y con cinco repeticiones en diseño de bloques completos al azar.

\section{Experimento 2. Comparación de vigor y rendimiento de semilla sexual de polinización libre producida en Nicaragua (La Almaciguera, 1991) y semilla producida por CIP e India.}

Este experimento se realizó en la finca "Los Cerrato", La Almaciguera (1350 msnm), Estelí; durante el periodo junio- setiembre de 1992. La SSP PL nacional fue producida en la finca "San Francisco", La Almaciguera (1350 msnm) en 1991; después de una evaluación preliminar de campo (en almácigos) se seleccionaron para la prueba comparativa 7 de las 13 progenies PL producidas: DTO-33, Serrana x LT-7, LT-7, Atzimba, CFK-69.1, DTO28 y 7XY. 1. Las progenies recibidas del CIP en 1992 fueron: LT-9 x TS-3, Atzimba x DTO-28, Serrana x 104-12LB, Achirana x 104-12LB, Serrana X LT-7, Atzimba x LT-7, HPS-7/13, LT-9 x 104-12LB, HPS-I/67, HPS-7/67, Maine-28 x 104-12LB, HPS-25/67, LT-9 x TPS-113, Maine-28 x TPS-113 y HPS-II/67. Las progenies procedentes de India fueron: HPS-25/67, HPS7/13, HPS-I/67, HPS- 7/67, HPS-II/13 y HPS-II/67. La evaluación se hizo en camas de crecimiento de $1.3 \mathrm{~m}$ de ancho y $0.2 \mathrm{~m}$ de alto utilizando suelo franco como sustrato de crecimiento, desinfectado con BASAMID (40 g/m2) y fertilización de $33.0 \mathrm{~g}$ de $\mathrm{N}, 83.0 \mathrm{~g}$ de $\mathrm{P}$ y 28.0 g de $\mathrm{K}$ por $\mathrm{m}^{2}$ en cinco 
fraccionamientos utilizando la fórmula fertilizante 12-30-10. Se utilizó unaregla marcadora para distanciamiento definido de $10 \times 10 \mathrm{~cm}$ entre un punto de siembra y otro con una profundidad de $0.5 \mathrm{~cm}$; se colocaron tres semillas por punto de siembra, la unidad experimental fue de $1 \mathrm{~m}^{2}$ en diseño BCA con cinco repeticiones. El vigor de la semilla fue medido con el Coeficiente de Velocidad (CoV), el porcentaje de sobrevivencia y materia seca de plántulas. El CoV (2) se evaluó por conteos diarios de emergencia de las hojas cotiledonales hasta diez días después de la siembra, el porcentaje de sobrevivencia y materia seca de plántulas fueron evaluados a los 30 días. La defoliación se hizo a los 90 días y se cosechó a los 105 días.

Experimento 3. Producción de semilla sexual híbrida en Nicaragua; La Almaciguera (1350 msnm; 1992) y el efecto de nitrógeno y fósforo adicional en las plantas madres sobre el vigor germinativo de la semilla.

Este experimento se realizó en la finca "San Francisco" en la localidad La Almaciguera (1350 msnm, $\left.12.8^{\circ} \mathrm{N}\right)$, Estelí durante el periodo juniosetiembre de 1992; los clones empleados fueron: Serrana INTA como parental femenino; LT-7 y 104-12LB como parentales masculinos. Estos clones provenientes del CIP tenían dos multiplicaciones y se sembraron en estado de brotación múltiple. Los masculinos se sembraron el 26 de mayo y Serrana el 2 de junio; la fertilización que recibieron al momento de la siembra fue de 150-375-0 kg/ha de N-P-K. El contenido de materia orgánica del suelo fue de 2.9\%, $21 \mathrm{ppm}$ de $\mathrm{P}$ (según método Bray), $\mathrm{pH}$ de 5.3 y su textura franca. Las variables en estudio fueron: 1) La capacidad de Serrana INTA para producir semilla híbrida con polen de LT-7 y 104-12LB en las condiciones locales y, 2) El efecto sobre el vigor germinativo de la semilla de la aplicación suplementaria de N y P juntos $(240 \mathrm{~kg} / \mathrm{ha}+600 \mathrm{~kg} / \mathrm{ha})$ durante el desarrollo de la semilla en comparación con la aplicación suplementaria simple de N (240 kg/ha). El diseño experimental fue de parcelas divididas con cuatro repeticiones, siendo las parcelas principales los cruzamientos Serrana x 104-12LB (A) y Serrana x LT-7 (B)- y las subparcelas los dos tipos de fertilización suplementaria $-\mathrm{N}$ (Fl) y N+P (F2)-. Se plantaron seis surcos de cada parental masculino de $43 \mathrm{~m}$ de longitud cada uno, $1.0 \mathrm{~m}$ de distancia entre surcos y $0.3 \mathrm{~m}$ entre tubérculos en una parcela adyacente al área experimental; los femeninos se plantaron en surcos de $10 \mathrm{~m}$ de longitud, distanciamiento de $1.5 \mathrm{~m}$ entre surcos, $0.4 \mathrm{~m}$ entre tubérculos con cuatro surcos para cada tratamiento. Las fertilizaciones suplementarias F1: N (urea) y F2: $\mathrm{N}+\mathrm{P}(18-46-0)$ a las plantas madre se hicieron en seis fraccionamientos cada 8 días, aplicado en banda a $10 \mathrm{~cm}$ de distancia de las plantas, a partir del momento en que se evidenció la presencia de los primeros botones florales; además se hicieron cuatro aplicaciones foliares de urea (2\% de concentración) para prolongar la senescencia de las plantas (12). Las plantas no fueron aporcadas y se sostuvieron con estacas y cuerdas para evitar el acamado y facilitar las polinizaciones. El polen obtenido de las flores de los clones LT-7 y 104-12LB se extrajo $12 \mathrm{hr}$ antes de ser usado mediante un vibrador, colocado en cápsulas de gelatina y almacenado sobre arroz tostado $\mathrm{a} \pm 10^{\circ} \mathrm{C}$ en un termo plástico con hielo. Las polinizaciones se hicieron entre las 6.30 am y las 11.30 am simultáneamente en los dos cruzamientos. 
Las condiciones de crecimiento caracterizadas por longitud de día de $13.5 \mathrm{hr}$ en los meses de junio-julio, temperatura ambiental promedio máx./mín. de 27 ${ }^{\circ} \mathrm{C} / 14.9{ }^{\circ} \mathrm{C}$, temperaturas promedio de suelo de $19.7{ }^{\circ} \mathrm{C}$ (a $20 \mathrm{~cm}$ de profundidad) y pp de $300 \mathrm{~mm}$ durante todo el período, fueron favorables para el desarrollo de las plantas; además que estuvieron libres de plagas y enfermedades. Las bayas se cosecharon entre las ocho y nueve semanas después del inicio de las polinizaciones, a la maduración completa de las plantas.

La semilla se extrajo sin fermentación de las bayas y desinfectada en una solución de hipoclorito de sodio al $0.5 \%$ por tres minutos; luego fue secada a $5 \%$ de humedad a $10{ }^{\circ} \mathrm{C}$ de temperatura durante 15 días en la cámara de secado de semillas del Departamento de Recursos Genéticos de la Universidad Nacional Agraria (UNA) de Managua; posteriormente se almacenó en termos con arroz tostado como desecante a temperatura ambiental promedio de $21^{\circ} \mathrm{C}$, cambiándolo continuamente.

El número de bayas y la cantidad de SSP producida por tratamiento se registró de las plantas de los surcos centrales y el peso de la semilla (peso de 100 SSP) de cada tratamiento se midió tomando 5 muestras de 100 semillas de cada uno utilizando una balanza analítica electrónica digital. A los 60, 75, 90 y 105 días de almacenamiento, fue evaluado el vigor germinativo de las semillas de todos los tratamientos en platos petri a temperatura ambiental $21.0{ }^{\circ} \mathrm{C}$, tomando muestras de 100 semillas con 7 repeticiones por tratamiento. La velocidad de germinación se evaluó con el CoV por conteos diarios de emisión de radícula de las semillas en un periodo de hasta diez días después de la siembra.

\section{RESULTADOSYDISCUSIÓN}

\section{Producción de semilla sexual de polinización libre (PL) en Nicaragua, La Almaciguera; 1300 msnm, 1991.}

Los resultados muestran que todos los clones parentales, excepto Atlantic, produjeron semilla PL y los rendimientos por planta se incrementaron significativamente respecto a la producción obtenida en 1988 en Jinotega (1330msnm) (21) y en Miniflor (1350 msnm) (23). Los clones 104.12 LB, Tollocan y 7XY. 1 produjeron la mayor cantidad de bayas por planta, pero sólo 104.12 LB y Tollocan produjeron más de 5.0 g de semilla por planta, no observándose relación entre el número bayas y la cantidad de semilla por planta. Los clones que tuvieron floración abundante y prolongada (Anexo 1) y que produjeron la mayor cantidad de semilla por planta como 104.12 LB y Tollocan tuvieron también la de menor peso (peso de 100 SSP) con baja capacidad de sobrevivencia en campo de la misma manera a lo observado con la producción y evaluación de la semilla PL del clon R128.6. Sin embargo, los clones que tuvieron el menor rendimiento de semilla (< de 1.0 g/planta) como DTO33, Serrana y CFK-69.1 tuvieron mejor sobrevivencia en campo; 
LT-7 y Atzimba fueron los clones de comportamiento intermedio en rendimiento de semilla y su vigor germinativo.

Algunas de las asociaciones de clones (femenino/masculino) como; LT7/Atlantic, Atzimba/Atlanticy DTO-28/Atlantic produjeron semilla de mejor comportamiento, en germinación y sobrevivencia en campo, que la semilla de los mismos clones asociados con otros como LT-7/Serrana, Atzimba/CFK69.1 y DTO-28/DTO-33 (Tabla 1). En la asociación Serrana INTA/LT-7 la totalidad de la semilla producida fue producto de cruzamiento natural por la actividad de los polinizadores, debido a que observaciones microscópicas constataron la inviabilidad del polen de Serrana. A pesar de la abundante floración de Serrana su producción de semilla sólo fue de 0.5 g por planta; sin embargo, el peso de 100 SSP (82.6 mg) fue similar al de la semilla del mismo cruce proporcionada por CIP $(86.0 \mathrm{mg})$.

La intensidad de la floración y fructificación de los clones parentales y la activa presencia de polinizadores por las favorables condiciones ambientales de precipitación, luz y temperatura durante el período mayo-agosto en la localidad de La Almaciguera, permitió determinar a este período del año como la época de plantación más adecuada para el uso de estos progenitores, identificar a esta zona como la más propicia para la producción de semilla de polinización libre o hibrida y caracterizar a cada progenitor en el área de producción de SSP; aspectos ecológicos claves en el avance del conocimiento tecnológico para producir SSP (18)particularmente en trópicos cálidos.

\section{Comparación de vigor y rendimiento de semilla sexual de polinización libre producida en Nicaragua (La Almaciguera, 1991) y semilla producida por CIP en Perú e India.}

Cuatro de las siete progenies PL locales tuvieron mayor vigor (CoV, sobrevivencia y materia seca de plántulas), que la mayoría de las progenies procedentes del CIP e India (Tablas 2, 3 y 4), pero similar a los híbridos CIP Serrana x LT-7, Atzimba x LT-7 y Atzimba x DTO-28 evaluados y seleccionados desde 1989 en Nicaragua. El rendimiento en kg y número de tubérculos $/ \mathrm{m}^{2}$ de las progenies PL Serrana/LT-7, LT-7, Atzimba y CFK-69.1 fue superior o similar al de las progenies de CIP e India. El carácter híbrido de la semilla local proveniente de la asociación Serrana/ LT-7 quedó evidenciado en su vigor y rendimiento significativamente mayor a todas las progenies evaluadas en este experimento a excepción de Serrana x LT-7 de CIP. Estos resultados de comparación entre semillas producidas en Nicaragua y por el CIP, concuerdan con los obtenidos en experiencias de años anteriores con semillas producidas localmente en 1988,1990 y 1991 (21,22,23).

Tabla 1. Producción de semilla y polinización libre en La Almaciguera, (1350 msnm); junio-setiembre 1991. 


\begin{tabular}{|c|c|c|c|c|c|}
\hline $\begin{array}{l}\text { Clones Parentales } \\
\text { femenino/masculino }\end{array}$ & $\begin{array}{c}\mathrm{N}^{\circ} \text { bayas } \\
\text { /plta. }\end{array}$ & $\begin{array}{l}\text { Sem./plta. } \\
\text { (g) }\end{array}$ & $\begin{array}{l}\text { Peso de } 100 \\
\text { Sem. (mg) }\end{array}$ & $\begin{array}{c}\text { Germ. } \% \\
\text { A }\end{array}$ & $\begin{array}{c}\text { Sobrev. \% } \\
\text { B }\end{array}$ \\
\hline 104-12LB/CFK-69.1 & 42.9 & 6.0 & $47.0 \mathrm{c}$ & 33.0 & $57.1 \mathrm{C}$ \\
\hline Tollocan/7XY.l & 46.1 & 5.1 & $45.7 \mathrm{c}$ & 39.4 & $64.6 \mathrm{C}$ \\
\hline 7XY.l/Tollocan & 47.2 & 2.7 & $68.8 \mathrm{~b}$ & 51.6 & $71.5 \mathrm{cb}$ \\
\hline LT-7/SerranaINTA & 26.7 & 2.6 & $67.2 \mathrm{~b}$ & 60.0 & $64.3 \mathrm{cb}$ \\
\hline LT-7/Atlantic & 23.3 & 2.0 & $69.1 \mathrm{~b}$ & 88.0 & 74.2 ba \\
\hline Atzimba/ Atlantic & 43.9 & 1.4 & $72.9 \mathrm{~b}$ & 85.0 & $71.5 \mathrm{cb}$ \\
\hline Atzimba/CFK-69.1 & 31.8 & 1.2 & $74.9 \mathrm{ab}$ & 23.0 & - \\
\hline DTO-28/Atlantic & 8.1 & 1.0 & $66.3 \mathrm{~b}$ & 68.4 & 72.8 ba \\
\hline DTO-28/DTO-33 & 8.5 & 1.0 & $68.2 \mathrm{~b}$ & 35.2 & 58.9 c \\
\hline CFK-69. 1/104 -12LB & 11.8 & 0.5 & $70.2 \mathrm{~b}$ & 33.4 & 74.2 ba \\
\hline Serrana INTA/LT-7 & 20.3 & 0.5 & $82.6 a$ & 37.0 & 75.8 ba \\
\hline CFK-69.1/Atzimba & 11.4 & 0.3 & $65.2 \mathrm{cb}$ & 52.2 & $70.8 \mathrm{cb}$ \\
\hline DTO33/DTO-28 & 2.9 & 0.2 & $66.4 \mathrm{~b}$ & 54.2 & $86.5 a$ \\
\hline Atlantic/DTO-28 & Insignific & & - & - & - \\
\hline Atlantic/Atzimba & Insignific. & & - & - & - \\
\hline Tukey $(\mathrm{p}=0.05)$ & & & 9.0 & & 15.2 \\
\hline
\end{tabular}


Tabla 2. Vigor y rendimiento de semilla PL producida en Nicaragua (Almaciguera; 1,350 msnm, 1991). Localidad de evaluación: La Almacigera; junio-setiembre 1992.

\begin{tabular}{|c|c|c|c|c|c|}
\hline \multirow{2}{*}{$\begin{array}{l}\text { Progenies } \\
\text { (PL) }\end{array}$} & \multirow{2}{*}{$\begin{array}{l}\text { Coef. de } \\
\text { veloc } \\
(\mathrm{CoV})^{*}\end{array}$} & \multirow{2}{*}{$\begin{array}{l}\text { Sobrev } \\
\text { plántula } \\
(\%)\end{array}$} & \multirow{2}{*}{$\begin{array}{l}\text { Mat. seca } \\
\text { plántula } \\
(\mathrm{g})^{*}\end{array}$} & \multicolumn{2}{|c|}{$\begin{array}{l}\text { Rendimiento en } \\
\text { almácigo }\end{array}$} \\
\hline & & & & $\overline{\left(\mathrm{kg} / \mathrm{m}^{2}\right)}$ & $\left(\mathrm{NTub} / \mathrm{m}^{2}\right)$ \\
\hline DTO-33 & 11.7 & $82.5 a$ & $0.22 \mathrm{ab}$ & $5.0 \mathrm{de}$ & $463.3 \mathrm{bc}$ \\
\hline Serrana/LT-7 & 11.8 & $81.5 a$ & $0.27 \mathrm{ab}$ & $9.1 \mathrm{a}$ & 678.8 a \\
\hline Atz.xLT-7(CIP) & 11.9 & 78.9a & $0.31 \mathrm{a}$ & $6.5 \mathrm{c}$ & $400.0 \mathrm{~cd}$ \\
\hline Serr.xLT-7(CIP) & 11.7 & $77.5 a$ & $0.28 \mathrm{ab}$ & $8.5 \mathrm{ab}$ & 480.0 bc \\
\hline LT-7 & 11.9 & $76.8 \mathrm{a}$ & $0.21 \mathrm{ab}$ & $6.7 \mathrm{c}$ & 520.0 bc \\
\hline Atzimba & 11.5 & $74.3 a$ & 0.16 bc & $6.1 \mathrm{~cd}$ & 535.0 bc \\
\hline CFK-69.1 & 11.5 & $59.0 \mathrm{~b}$ & $0.16 \mathrm{bc}$ & $7.4 \mathrm{bc}$ & $563.8 \mathrm{ab}$ \\
\hline DTO-28 & 11.3 & $58.5 \mathrm{~b}$ & 0.18 bc & $4.2 \mathrm{ef}$ & 270.3 de \\
\hline 7XY.1 & 11.4 & $23.8 \mathrm{c}$ & $0.08 \mathrm{c}$ & $3.0 \mathrm{f}$ & $220.0 \mathrm{e}$ \\
\hline Tukey $(\mathrm{p}=0.05)$ & 0.5 & 14.3 & 0.13 & 1.5 & 138.1 \\
\hline
\end{tabular}

* Evaluación a los 30 días después de la siembra.

Tabla 3. Vigor y rendimiento de semilla sexual de CIP evaluada en La Almacigera; junio-setiembre 1992.

\begin{tabular}{|c|c|c|c|c|c|}
\hline \multirow{2}{*}{$\begin{array}{l}\text { Progenies } \\
\text { veloc. }\end{array}$} & \multirow{2}{*}{$\begin{array}{l}\text { Coef. } \\
\text { Veloc } \\
(\mathrm{CoV})^{*}\end{array}$} & \multirow{2}{*}{$\begin{array}{c}\text { Sobrev. } \\
\text { Plántula } \\
\text { (\%)* }\end{array}$} & \multirow{2}{*}{$\begin{array}{c}\text { Mat. seca } \\
\text { en almácigo } \\
(\mathrm{g})^{*}\end{array}$} & \multicolumn{2}{|c|}{ Rendimiento } \\
\hline & & & & $\left(\mathrm{kg} / \mathrm{m}^{2}\right)$ & $\left(\mathrm{NTub} / \mathrm{m}^{2}\right)$ \\
\hline LT-9xTS-3 & 7.9 & 76.3a & 0.14 abc & $6.4 \mathrm{ab}$ & 353 \\
\hline Atz. x DTO-28 & 8.0 & $64.0 \mathrm{ab}$ & $0.22 \mathrm{a}$ & $5.9 \mathrm{ab}$ & 440 \\
\hline Serr.xl04-12LB & 8.0 & $61.3 \mathrm{abc}$ & $0.14 \mathrm{abc}$ & $4.8 \mathrm{ab}$ & 253 \\
\hline Achr.xl04-12LB & 7.9 & $59.7 \mathrm{abc}$ & $0.11 \mathrm{bc}$ & $6.4 a b$ & 380 \\
\hline HPS-7/13 & 8.0 & $57.0 \mathrm{bc}$ & $0.14 a b$ & $6.7 \mathrm{ab}$ & 347 \\
\hline LT-9x 104-12LB & 7.9 & $50.3 \mathrm{bc}$ & 0.08 bc & $7.8 \mathrm{a}$ & 325 \\
\hline HPS-I/67 & 7.9 & $49.7 \mathrm{bc}$ & 0.11 bc & $3.7 \mathrm{~b}$ & 297 \\
\hline HPS-7/67 & 7.9 & 46.7 bcd & $0.12 \mathrm{abc}$ & $5.8 \mathrm{ab}$ & 350 \\
\hline Maine28xl04.12LB & 7.9 & $44.0 \mathrm{~cd}$ & $0.12 \mathrm{abc}$ & $5.1 \mathrm{ab}$ & 303 \\
\hline HPS-25/67 & 7.9 & $43.0 \mathrm{~cd}$ & $0.15 \mathrm{ab}$ & $5.7 \mathrm{ab}$ & 298 \\
\hline LT-9xTPS-113 & 7.8 & $30.3 \mathrm{~d}$ & $0.11 \mathrm{bc}$ & $6.5 \mathrm{ab}$ & 282 \\
\hline Maine28xTPS113 & 7.8 & 29.0 de & $0.07 \mathrm{bc}$ & 5. $1 \mathrm{ab}$ & 238 \\
\hline HPS-II/67 & 7.5 & $13.3 \mathrm{e}$ & $0.04 \mathrm{c}$ & $6.8 \mathrm{ab}$ & 268 \\
\hline Tukey(p=0.05) & 0.3 & 18.7 & 0.11 & 3.1 & ns \\
\hline
\end{tabular}

* Evaluación a los 30 días después de la siembra.

Tabla 4. Vigor y rendimiento de semilla sexual de India evaluada en La 
Almacigera; junio-setiembre 1992.

\begin{tabular}{|c|c|c|c|c|c|}
\hline \multirow[t]{2}{*}{$\begin{array}{l}\text { Progenies } \\
\text { veloc }\end{array}$} & $\begin{array}{l}\text { Coef. } \\
\text { veloc. }\end{array}$ & $\begin{array}{l}\text { Sobrev. } \\
\text { Plántula }\end{array}$ & $\begin{array}{l}\text { Mat. seca } \\
\text { en almacigo }\end{array}$ & \multicolumn{2}{|c|}{ Rendimiento } \\
\hline & $(\mathrm{CoV})^{*}$ & $(\%)^{*}$ & $(\mathrm{~g})^{*}$ & $\left(\mathrm{~kg} / \mathrm{m}^{2}\right)$ & $\left(\mathrm{NTub} / \mathrm{m}^{2}\right)$ \\
\hline PS-25/67 & 7.8 & $63.8 \mathrm{a}$ & $0.12 \mathrm{ab}$ & $6.5 a$ & $393 a b$ \\
\hline PS-7/13 & 7.8 & 61.8a & $0.10 \mathrm{~b}$ & $5.3 \mathrm{ab}$ & $313 \mathrm{ab}$ \\
\hline PS-I/67 & 7.9 & $60.3 a$ & $0.19 a$ & $5.7 \mathrm{ab}$ & $341 \mathrm{ab}$ \\
\hline PS-7/67 & 7.9 & $58.5 a$ & $0.14 \mathrm{ab}$ & 7.3a & $473 \mathrm{a}$ \\
\hline PS-II/13 & 7.7 & $45.0 \mathrm{ab}$ & $0.18 \mathrm{a}$ & $3.7 \mathrm{~b}$ & $204 \mathrm{~b}$ \\
\hline PS-II/67 & 7.7 & $25.0 \mathrm{~b}$ & $0.06 \mathrm{~b}$ & $3.0 \mathrm{~b}$ & $253 \mathrm{~b}$ \\
\hline ukey $(p=0.05)$ & 0.2 & 23.6 & 0.08 & 3.0 & 195 \\
\hline
\end{tabular}

* Evaluación a los 30 días después de la siembra.

Estos resultados no contradicen la comprobada superioridad de la semilla hibrida sobre la de PL $(6,7,8)$ sino, muestran la diferencia en la expresión del vigor de semillas producidas en dos ambientes distintos por efecto del clima cálido en que crecen; es decir, los híbridos producidos a temperaturas bajas o moderadas son más sensibles a las temperaturas elevadas que aquellas semillas producidas en el mismo ambiente cálido en que se siembran $(9,10)$.

Bajo condiciones de campo, con variaciones diarias de temperatura mín/máx de $15{ }^{\circ} \mathrm{C} / 27{ }^{\circ} \mathrm{C}$, irregular suministro de agua por precipitaciones y uso de suelo como sustrato de almácigos; los porcentajes de sobrevivencia y CoV del conjunto de las progenies evaluadas -Nicaragua, CIP e India- fueron bajos (Tablas 2,3 y 4) en relación a los valores establecidos como aceptables para SSP de buen vigor (germinación $>90 \%$ y CoV >20) (2); esto indica que la SSP actualmente disponible, incluidos los híbridos de mayor aceptación por los productores, como Serrana x LT-7 y Atzimba x LT-7, tienen bajo vigor al crecer en condiciones de campo en ambientes de clima cálido como Nicaragua, Sin embargo, por lo observado en estas experiencias, aún utilizando parentales no adaptados completamente para producir semilla en los trópicos, la semilla obtenida ha mostrado buen potencial de adaptación y rendimiento en las condiciones ecológicas que el productor las va emplear; el uso de parentales mejor adaptados y el correcto almacenamiento realzarán la calidad de la semilla producida.

\section{Producción de semilla sexual híbrida en Nicaragua; La Almaciguera (1350 msnm; 1992) y el efecto de nitrógeno y fósforo adicional en las plantas madre sobre el vigor germinativo de la semilla.}

El efecto positivo de las altitudes comprendidas entre los 1300 y $1400 \mathrm{msnm}$ sobre el peso y vigor de la semilla PL de la variedad Désirée observado en experimentos durante 1990 (22) apoyan lo propuesto por Malagamba (10) sobre el efecto compensatorio de la altitud cuando se produce SSP en trópicos cálidos como Nicaragua. La intensa floración de Serrana INTA y de los parentales masculinos LT-7 y 104-12LB, confirmaron las observaciones del año anterior 
sobre las favorables condiciones ambientales de la zona de La Almaciguera durante el periodo mayo-setiembre para la producción de semilla sexual $(22,23)$ (Figura 1). La distribución e intensidad de las precipitaciones durante el periodo de desarrollo de las plantas mantuvo la humedad del suelo a capacidad de campo y temperaturas inferiores a $20{ }^{\circ} \mathrm{C}$ en la zona radicular (25 cm de profundidad de la superficie del suelo) que favorecen el desarrollo de la parte aérea $(19,4)$ y particularmente la floración evitando la senescencia temprana; la regularidad de las precipitaciones, durante las tardes y noches, facilitó las polinizaciones. A diferencia del año anterior, la senescencia de las plantas en este experimento se pudo prolongar hasta 105 días, por las fertilizaciones foliares de $\mathrm{N}$ con urea (12) y las bayas se cosecharon con mayor madurez.

Figura 1 Temperatura del aire (máx/min), del suelo y precipitación durante la producción de SSP hibrida; La Almaciguera 1,300m. 1992

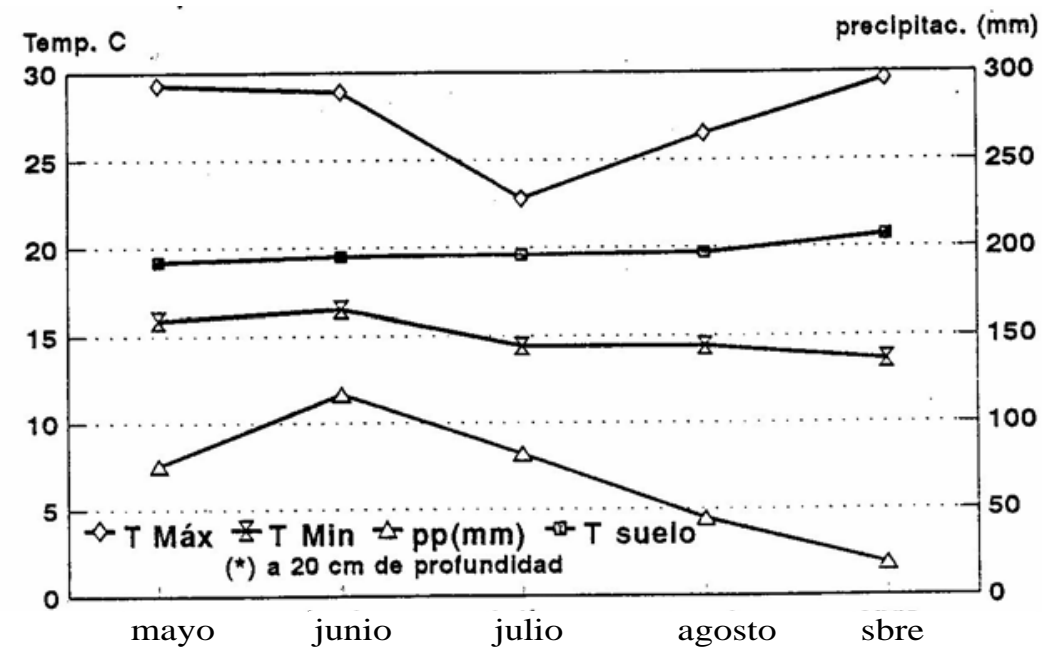

\begin{tabular}{|c|c|c|c|c|c|}
\hline T Max & 29.3 & 28.9 & 22.8 & 26.5 & 29.6 \\
\hline T Min & 15.9 & 16.5 & 14.4 & 14.4 & 13.6 \\
\hline $\mathrm{pp}(\mathrm{mm})$ & 75.5 & 116.5 & 82 & 44.5 & 18.5 \\
\hline T suelo & 19.2 & 19.5 & 19.6 & 19.7 & 20.7 \\
\hline
\end{tabular}


La producción total de SSP híbrida en los $1032 \mathrm{~m}^{2}$ del experimento fue de 7.1 kg y $2.46 \mathrm{~kg}$ de Serrana x LT-7 y $4.66 \mathrm{~kg}$ de Serrana x 104.12LB. Los resultados obtenidos en este año contrastan significativamente con los del año 1988 (21) en que se obtuvieron bajísimas cantidades de semilla -menores de 0.5 g/planta- como consecuencia de la inadecuada elección de la altitud y época de plantación (22); lo que confirma también, las investigaciones de INIA-Chile acerca de que la plantación de los parentales en la época apropiada es el método más conveniente para mejorar la eficiencia de producción de SSP híbrida $(3,18)$.

La producción de polen del clon 104.12LB fue mayor a la de LT-7, aunque ambos aportaron cantidades suficientes para la polinización; sin embargo, los rendimientos en bayas y semilla por planta del cruzamiento Serrana $\mathrm{x}$ 104.12LB (B), fueron significativamente mayores a los del cruzamiento Serrana x LT-7 (A) (Tabla 5), lo que indica que el polen de 104.12LB tuvo mejor conservación de su fertilidad que el de LT-7.

Las aplicaciones de $\mathrm{N}$ y $\mathrm{N}+\mathrm{P}$ adicionales no tuvieron efecto sobre el establecimiento de bayas, rendimiento de semilla por planta ni en el peso de la semilla en ninguno de los cruzamientos; pero al evaluar el vigor germinativo de la semilla a los 60, 75, 90 y 105 días de almacenamiento (dda), se registró que las semillas de las plantas madre que recibieron $\mathrm{N}+\mathrm{P}$ (F2) adicional tuvieron valores de $\mathrm{CoV}$ y porcentaje de germinación significativamente superiores, en ambos cruzamientos, que las semillas de las plantas madre que recibieron sólo $\mathrm{N}$ adicional (Fl) (Tabla 5). Estos resultados soportan lo propuesto por Quick (17) de que la nutrición previa de la planta madre con P tiene influencia sobre la habilidad de la semilla para iniciar la germinación y optimiza su producción $(9,16)$. En las evaluaciones a los 60 y 75 días, debido a que la germinación inició al sexto día, los conteos se hicieron hasta el undécimo y décimo día respectivamente, en que se estabilizó la germinación; pero en la tercera y cuarta evaluación, en que la germinación inició al tercer día, los conteos se hicieron hasta el octavo y sexto día en que los tratamientos AF2 y BF2 alcanzaron sus máximos valores; mientras AF1 y BF1 aún no lo hacían, lo que indica la rápida pérdida del reposo de las semillas de ambos cruzamientos por efecto del $\mathrm{P}$ adicional y la rápida reducción del periodo entre el inicio y fin de la germinación, de seis a cuatro días (Tabla 5).

El peso de la semilla -correlacionado positivamente con el vigor (13)- de los cruzamientos locales es significativamente menor que el de la semilla proporcionada por CIP (Serrana x LT-7 producida en Osorno, Chile y Serrana x 104.12LB); pues la semilla producida en condiciones tropicales es más liviana que aquella producida en condiciones óptimas $(10,13,14)$; sin embargo, las semillas locales mostraron mejor $\mathrm{CoV}$, al iniciar germinación al tercer día; mientras las de CIP lo hicieron al quinto; aunque el porcentaje final de germinación fue similar en ambas (Tabla 5). 
Tabla 5. Producción de SSP hibrida y el efecto de $N$ y $P$ adicional en su vigor germinativo. La Almaciguera, 1350 msnm, junio-setiembre 1992.

\begin{tabular}{|c|c|c|c|c|c|c|c|c|c|c|c|c|}
\hline \multirow[b]{2}{*}{ (C) } & \multirow{2}{*}{$\begin{array}{l}\text { NP } \\
\text { Adic. } \\
\text { (F) }\end{array}$} & \multirow{2}{*}{$\begin{array}{c}\mathrm{N}^{\circ} \\
\text { Bayas } \\
\text { planta }\end{array}$} & \multirow{2}{*}{$\begin{array}{c}\text { Sem./ } \\
\text { planta } \\
\text { (g) }\end{array}$} & \multirow{2}{*}{$\begin{array}{c}\text { Peso } \\
100 \\
\text { Sem. } \\
\text { (mg) }\end{array}$} & \multicolumn{4}{|c|}{$\begin{array}{c}\text { Coefic. velocidad } \\
\text { (CoV) }\end{array}$} & \multicolumn{4}{|c|}{$\begin{array}{c}\text { Germinación } \\
\text { (\%) }\end{array}$} \\
\hline & & & & & 1ra & 2da & 3ra & 4 ta & 1ra & 2da & 3ra & 4 ta \\
\hline \multirow[t]{2}{*}{ A } & $\mathrm{Fl}$ & 35.8a & 3.62a & 70.6 & 10.0 & 11.8 & 15.1 & 19.7 & 37.0 & 85.0 & 68.4 & 87.3 \\
\hline & $\mathrm{F} 2$ & $40.0 \mathrm{a}$ & 3.36a & 71.5 & 9.9 & 12.0 & 15.9 & 20.7 & 55.0 & 98.2 & 97.9 & 98.7 \\
\hline \multirow[t]{2}{*}{$\mathrm{B}$} & $\mathrm{Fl}$ & $29.9 b$ & $1.88 \mathrm{~b}$ & 72.7 & 9.7 & 11.5 & 15.3 & 19.2 & 23.8 & 65.4 & 69.1 & 76.8 \\
\hline & $\mathrm{F} 2$ & $27.4 \mathrm{~b}$ & $2.07 \mathrm{~b}$ & 71.9 & 9.8 & 11.8 & 16.4 & 20.5 & 33.4 & 86.2 & 95.0 & 94.6 \\
\hline \multirow{3}{*}{$\begin{array}{l}\text { Tuke } \\
\text { CxF }\end{array}$} & $(\mathrm{p}=0.05)$ & 5.0 & 1.15 & ns & ns & 0.14 & 0.15 & 0.4 & 5.9 & 6.8 & 4.8 & 5.1 \\
\hline & & ns & ns & $\mathrm{ns}$ & ns & ns & ns & ns & ns & ns & ns & ns \\
\hline & & & & Peso & & $\mathrm{CoV}$ & & & & Germ. & & \\
\hline \multicolumn{3}{|c|}{ Serrana xLT-7(CIP) } & & 86.0 & & 14.4 & & & & 94.5 & & \\
\hline \multicolumn{3}{|c|}{ Serrana x104-12LB(CIP) } & & - & & 14.9 & & & & 93.4 & & \\
\hline
\end{tabular}

(C: Cruzamiento: A = "Serrana\104.12LB"; B= "SerranaxLT-7".

F1: $240 \mathrm{~kg} \mathrm{~N}$ adicional; F2: $240 \mathrm{~kg} \mathrm{~N}$ adicio+600 kg P adic.

$1 \mathrm{ra}=60 ; 2 \mathrm{da}=75 ; 3 \mathrm{ra}=90 ; 4 \mathrm{ta}=105$ días de almacenamiento.

Producción Total en 1,032 $\mathrm{m}^{2}$ : Serrana x 104-12LB $=4.66 \mathrm{~kg}$

Serrana $\mathrm{x}$ LT-7 $=2.46 \mathrm{~kg}$

El rendimiento de semilla por planta y la producción total obtenida fue mermada por desperfecto del vibrador extractor de polen que obligó a detener las polinizaciones 18 días antes del fin de la floración de Serrana, lo cual incrementó el costo de la semilla producida. Aún así, los $7.1 \mathrm{~kg}$ de semilla, cubrirán las necesidades actuales del país. El costo de producción de 1 kg de Serrana x LT-7 fue de \$ 750 dólares y el de Serrana x 104.12LB fue de \$ 400(Anexo 2).

Los resultados generales obtenidos muestran el gran potencial de algunas microzonas ubicadas entre los 1300 y 1400 msnm del trópico cálido de Nicaragua para la producción de SSP cuando se emplean clones adaptados a estas regiones y más aún si se proyecta en el futuro cercano la utilización de materiales genéticos y combinaciones de parentales que permitan una mejor posibilidad de producir semilla de híbridos mejorados, bajo condiciones ecológicas similares a las presentes en las zonas tropicales que demandan el uso de esta tecnología (11). De otra parte, la adecuada nutrición de las plantas madres con $\mathrm{N}$ y $\mathrm{P}$ y la correcta manipulación de la semilla en su almacenamiento (14) permitirán superar las barreras actuales que limitan la producción de SSP en los trópicos. 


\section{AGRADECIMIENTOS}

Expresamos nuestro sincero agradecimiento al Sr. José Dávila por su valiosa participación en la ejecutoria de los experimentos en 1991, al Sr. Cristóbal Cruz propietario de la finca "San Francisco" quien por dos años consecutivos colaboró activamente en la realización de los experimentos de producción de SSP y a los Srs. Gustavo, Matias y Eriberto Cerrato propietarios de la finca "Los Cerrato", por su cooperación en las evaluaciones de SSP de Nicaragua, CIP e India.

\section{REFERENCIAS BIBLIOGRÁFICAS}

1. Centro Internacional de la Papa. 1988. Informe Anual CIP 1986-87. Lima, Perú. 210 p.

2. Centro Internacional de la Papa. 1989. Informe Anual CIP 1988. Lima, Perú. 226 p.

3. Centro Internacional de la Papa. 1992. Informe Anual CIP 1991. Lima, Perú. 168 p.

4. Christiansen, M; Lewis, Ch. 1987. Mejoramiento de plantas en ambientes desfavorables. Edit. LIMUSA, México. 534 p.

5. Golmirzaie, A.M.; Mendoza, H. 1988. Estrategias de Mejoramiento para la Producción de Semilla Sexual de Papa. CIP Circular. Centro Internacional de la Papa.vol 16.N14.

6. Golmirzaie, A.M.; Scrquén F.; Ortiz, R. 1990. Evaluación de tres progenies de polinización en seis progenies de la papa (Solanum tuberosum L.) provenientes de semilla (sexual). Revista Latinoamericana de la Papa. 3:13-19.

7. Golmirzaie, A.; Ortiz, R; Serquén, F. 1990. Genética y Mejoramiento de la Papa mediante semilla (sexual). Centro Internacional de la Papa. Lima, Perú. 35 p.

8. Kidane-Mariam,HM; Mendoza, R A.; Wissar,RO. 1985. Performances of true potato seed families derived from intermating tetraploid parental lines. Am. Potato J. 62:643-652. 
9. Malagamba, P. 1983. Some practical considerations on the production of TPS. True Potato Seed (TPS) Letter. International Potato Center (CIP), vol 4 NI. Lima, Perú.

10. Malagamba, P. 1988. Potato Production of True Seed in Tropical Climates. Hort. Sciences; vol.23(3):495-500.

11. Malagamba, P; Cabello, R. 1991. Producción de semilla sexual en diferentes ambientes p.21-25. Semilla Sexual en Latinoamérica. Centro Internacional de la Papa. Lima, Perú. 86 p.

12. Midmore, D. 1989. Agronomía para la producción de papa en climas cálidos. Guía de Investigación CIP 9. Centro Internacional de la Papa. Lima, Perú. 48 p.

13. Pallais, N; Villagarcía, S.; Fong, N.; Tapia, J.; García, R. 1987. Effect of Suplemental Nitrogen on True Potato Seed Weight. Am. Potato J. 64:483-491.

14. Pallais, N. 1987. True Potato Seed Quality. Theor. Appl. Genet. 73:784-792.

15. Pallais, N; Falcón, R 1991. Calidad de semilla y manejo en poscosecha p.11-20. Semilla Sexual de Papa en Latinoamérica. Centro Internacional de la Papa. Lima, Perú $86 \mathrm{p}$.

16. Potash and Phosphate Institute of Canada and Foundation for Agronomic Research. 1988. Manual de Fertilidad de Suelos. 82p.

17. Quick, W.A.; Hsiao, A.I.; Jain, J.C. 1983. Endogenus inorganic phosphate in relation to seed dormancy and germination of wilt oasis. Plant Science Letters, 28:129135.

18. Rojas, J.S. 1991. Avances en la producción de semilla sexual híbrida de papa (SSP) en Chile, p.47-57. Semilla Sexual de Papa en Latinoamérica. Centro Internacional de la Papa. Lima, Perú. 86 p.

19. Sattelmacher, B. 1984. Physiological Aspects of the Adaptation of the Potato to the Hot Humid Tropics, p.465- 469. En: Sixth Symposium of the International Society for Tropical Roots Crops. 1983. International Potato Center (CIP). Lima, Perú.

20. Soffer, H.; Smith. D.E. 1974. Studies on Lettuce Seed Quality: V. Nutritional Effects. J. Amer. Soc. Hort. Sci. 99(5):459-463.

21. Torres, F; Gonzales, C.; Tórrez, C. 1990. Perspectivas del uso de la semilla (sexual) de papa en Nicaragua Revista Latinoamericana de la Papa. 3:41-55.

22. Torres, F; Gonzales, C.; Tórrez, C. 1991. Semilla Sexual en la Producción de Papa en Nicaragua, p. 59-70. Semilla Sexual de Papa en Latinoamérica. Centro Internacional de la Papa. Lima, Perú. 86 p. 
23. Torres, F. 1992. Semilla Sexual (Botánica): Una Tecnología Sostenible en la Producción de Papa en Nicaragua. En: II Congreso sobre Generación y Transferencia de Tecnología Agropecuaria; 22 al 24 de Abril de 1992. Universidad Nacional Agraria. Managua. Nicaragua.

24. Yagodin, B.A. Agroquímica I. Edit. MIR Moscú. 416 p. 
Anexo 1: Información sobrapadres potenciales y progenies de semilla(sexual).

Tabulación de caracteres reproductivos.

Fecha de siembra: 23/5/91

\begin{tabular}{lllllllllllll}
\hline CLON & 4 & 5 & 6 & 7 & 8 & 9 & 10 & 11 & 12 & $12^{*}$ & $13^{*}$ & 14 \\
\hline Tollocan & $30 / 6$ & 7 & 9 & 5 & 5 & 2 & 2 & 5 & 7 & 46.1 & 5.1 & \\
7XY1 & $29 / 6$ & 7 & 7 & 5 & 5 & 2 & 2 & 4 & 7 & 47.2 & 1.7 & \\
LT-7 & $24 / 6$ & 7 & 7 & 5 & 5 & 2 & 2 & 5 & 7 & 26.7 & 2.5 & \\
Serrana & $24 / 6$ & 9 & 9 & 5 & 5 & 2 & 2 & 3 & 7 & 20.2 & 0.5 & a \\
Atlantic & $22 / 6$ & 5 & 5 & 5 & 4 & 1 & 1 & 0 & 0 & 0.56 & 0.0 & \\
Atámba & $25 / 6$ & 7 & 9 & 5 & $5-$ & 2 & 2 & & 7 & 43.8 & 1.4 &
\end{tabular}

14: a = La última floración produjo mayor cantidad de bayas que las primeras. Las flores fueron escasamente visitadas por los polinizadores.

Fecha de siembra: 7/6/91

\begin{tabular}{lllllllllllll}
\hline CLON & 4 & 5 & 6 & 7 & 8 & 9 & 10 & 11 & 12 & $12^{*}$ & $13^{*}$ & 14 \\
\hline DTO-28 & $10 / 7$ & 7 & 5 & 5 & 4 & 2 & 2 & 3 & 5 & 8.3 & 1.0 & $\mathrm{~b}$ \\
DTO-33 & $11 / 7$ & 3 & 3 & 3 & 4 & 2 & 2 & 1 & 3 & 2.9 & 0.2 & $\mathrm{~b}$ \\
CFK-69.1 & $20 / 7$ & 5 & 7 & 5 & 5 & 2 & 2 & 5 & 5 & 11.8 & 0.4 & \\
104-12LB & $12 / 7$ & 9 & 7 & 5 & 4 & 2 & 2 & 5 & 7 & 49.2 & 60 & \\
\hline
\end{tabular}

12* : Bayas PL/planta

13* : Gramos de semilla/planta

14: $\mathrm{b} \quad=$ Apariencia virósica plantas pequeñas.

Comentario General: Los tubérculos empleados se encontraban en brotación múltiple e Inicio de envejecimiento (deshidratación).

Lugar y Altitud: La Almaciguera. Esteli; $12^{\circ}$ 80' LN; 1,350 msnm. Periodo: junio -setiembre de 1991.

Temp. med. máx./min: $27{ }^{\circ} \mathrm{C} / 17{ }^{\circ} \mathrm{C}$; Precipitac. jun-set: $250 \mathrm{~mm}$ otra información climatológica: Fuertes vientos a partir del 15 de agosto, e inicio de escases de lluvias.

Los códigos de las tablas corresponden al formato propuesto en "Genética y Mejoramiento de la Papa Mediante Semilla Sexual. Golmirzaie, A.; Ortiz, R.; Serquén,F. 1991. CIP. Lima. Perú. 
Columna 1

Columna 2

Columna 3

Columna 4

Columna 5 Intensidad de la floración (tomada 10 dias después de la FIF)
0 . Ausencia de botones
5. Floración moderada
1. Botones abortados
7. Floración abundante
3. Floración escasa
9. Floración muy abundante

Columna 6
1. Muy corta
3. Corta
5. Media
7. Larga
9. Muy larga
5 días
5 a 10 días
10 a 20 días
20 a 30 días
30 a 40 dos

(días después de FIF)

Columna 7 Número de flores por inflorescencia (10 días después de FIF)
Columna 8
Columna 9
Longitud del estilo
1. Más corto que la columna estaminal y en forma de $S$
2. Más corto que la columna estaminal y derecho
3. Igual a la columna estaminal
4. Ligeramente más largo que la columna estaminal
5. Más largo que la columna estaminal
7. Mucho más largo que la columna estaminal
Tipo de antera
1. Anteras distorsionadas
2. Anteras normales

1. Una sola

3. Pocas (2-5)

5. Varias (6-10)

7. Muchas (más de 20)

Columna $10 \quad$ ¿Las flores atraen abejas?

1. Si

2. No

Columna 11 Producción de polen
0 . Nada
1. Poco
3 Promedio
5 Abundante

Columna 12 Número de frutos de polinización libre (logrados por planta)
0 . Ninguno
3. Pocos
(0)
5. Varios
7. Muchos
(más de 50)

Columna 13 Número de semillas de polinización libre logradas (por baya)

$\begin{array}{ll}\text { 0. Ninguna } & (0) \\ \text { 3. Pocas } & (1-50) \\ \text { 5. Varias } & (50-100) \\ \text { 7. Muchas } & (100-200) \\ \text { 9. Abundantes } & \text { (más de 200) }\end{array}$

Columna 14

Anote las enfermedades, el tamaño de tubérculo, la edad fisiológica de los tubérculos, u otros factores que puedan afectar la floración o la formación de la semilla. Si se efectúan pruebas de germinación de polen, anote los resultados, como porcentaje, en esta columna. 
Anexo 2. Costo de producción de SSP híbrida en $1032 \mathrm{~m}^{2}$

\begin{tabular}{|c|c|c|c|c|}
\hline \multirow{2}{*}{\multicolumn{2}{|c|}{ CONCEPTO }} & \multicolumn{3}{|c|}{ COSTO } \\
\hline & & $\begin{array}{l}\text { Córdoba oro } \\
\text { (C\$) }\end{array}$ & $\begin{array}{l}\text { Dólares } \\
\text { (US\$) }\end{array}$ & $\%$ \\
\hline Implen & mentos + renta de tierra & 400.0 & 80.0 & 2.2 \\
\hline Multip & licación de parentales & 400.0 & 80.0 & 2.2 \\
\hline Agroqu & uímicos & 842.5 & 168.7 & 4.6 \\
\hline & Fertilizantes & 257.5 & 51.5 & \\
\hline & Herbicidas & 30.0 & 6.0 & \\
\hline & Insecticidas & 227.5 & 45.5 & \\
\hline & Fungicidas & 230.0 & 46.0 & \\
\hline & Desinfectantes & 29.5 & 5.9 & \\
\hline & Adherente & 69.0 & 13.8 & \\
\hline Mano & de Obra & $3,036.0$ & 607.2 & 16.6 \\
\hline & Preparación terreno, siembra y manejo & 727.0 & 145.4 & \\
\hline & Colocación estacas/cuerdas & 121.0 & 24.2 & \\
\hline & Extracción de polen & 454.5 & 90.9 & \\
\hline & Polinización & $1,442.5$ & 288.5 & \\
\hline & Cosecha bayas/extracc. Semilla & 242.5 & 48.5 & \\
\hline & Cosecha parentales & 48.5 & 9.7 & \\
\hline Materi & iales & $1,125.0$ & 225.0 & 6.1 \\
\hline Combu & ustibles & $2,220.0$ & 444.0 & 12.1 \\
\hline Secado & o de semilla & 227.5 & 45.5 & 1.2 \\
\hline Salario & o técnico & $7,270.0$ & $1,454.0$ & 39.7 \\
\hline Transp & oorte & $1,130.0$ & 226.0 & 6.2 \\
\hline Gastos & administrativos & $1,849.0$ & 333.0 & 9.1 \\
\hline Total & & $18,317.0$ & $3,663.4$ & 100.0 \\
\hline Costo & $\begin{array}{l}2.46 \text { kg Serrana x LT-7 }\left(516 \mathrm{~m}^{2}\right) \\
4.66 \mathrm{kgSerr} . \backslash 104-12 \mathrm{LB}(516 \mathrm{~m} 2)\end{array}$ & & $\begin{array}{l}1,831.7 \\
1,831.7\end{array}$ & \\
\hline & $\begin{array}{l}1.0 \mathrm{~kg} \text { Serrana x LT-7 } \\
1.0 \mathrm{~kg} \text { Serrana x } 104-12 \mathrm{LB}\end{array}$ & & $\begin{array}{l}744.6 \\
393.1\end{array}$ & \\
\hline
\end{tabular}

Tasa de cambio 1992 US\$ x 5.0 Córdoba oro C\$ 